\title{
Investigation of Two-Photon Absorption Properties in Branched Alkene and Alkyne Chromophores
}

\author{
Ajit Bhaskar, ${ }^{\dagger}$ Guda Ramakrishna, ${ }^{\dagger}$ Zhikuan Lu, $^{\dagger \dagger}$ Robert Twieg, ${ }^{\dagger \dagger}$ Joel M. Hales, ${ }^{\dagger \dagger}$ \\ David J. Hagan, ${ }^{\dagger \dagger}$ Eric Van Stryland, ${ }^{\dagger \dagger}$ Theodore Goodson III $^{\dagger *}$ \\ ${ }^{\dagger}$ Department of Chemistry, Department of Macromolecular Science and Engineering, \\ University of Michigan, Ann Arbor, MI, 48109 \\ ${ }^{\dagger \dagger}$ Department of Chemistry, Kent State University, Kent Ohio 44242 \\ ${ }^{+\dagger}$ School of Optics/CREOL, University of Central Florida, Orlando, FL, 32816. \\ *E-mail: tgoodson@umich.edu
}

\section{Supporting information}

\section{Complete references of $1(a)$ and 1(b)}

1(a). Cumpston, B. H.; Ananthavel, S. P.; Varlow, S.; Dyer, D. L.; Ehrlich, J. E.; Erskine, L. L.; Heikal, A. A.; Kuebler, S. M.; Sandy Lee, I. -Y.; McCord-Maughon, D.; Qin, J.; Roeckel, H.; Rumi, M.; Wu, X.-L.; Marder, S. R.; Perry, J. W. Nature 1999, 398, 51.

1(b). Albota, M.; Beljonne, D.; Bredas, J.-L.; Ehrlich, J. E.; Fu, J.-Y.; Heikal, A. A.; Hess, S. E.; Kogej, T.; Levin, M. D.; Marder, S. R.; McCord-Maughon, D.; Perry, J. W.; Rockel, H.; Rumi, M.; Subramaniam, G.; Webb, W. W.; Wu, X.-L.; Xu, C. Science 1998, $281,1653$. 